\title{
Skew Pieri Rules for Hall-Littlewood Functions
}

\author{
Matjaž Konvalinka $1 \|$ and Aaron Lauve $\equiv^{2}$ \\ ${ }^{1}$ Dept. of Mathematics, University of Ljubljana, Slovenia \\ ${ }^{2}$ Dept. of Mathematics and Statistics, Loyola University Chicago, USA
}

\begin{abstract}
We produce skew Pieri Rules for Hall-Littlewood functions in the spirit of Assaf and McNamara (FPSAC, 2010). The first two were conjectured by the first author (FPSAC, 2011). The key ingredients in the proofs are a $q$-binomial identity for skew partitions that are horizontal strips and a Hopf algebraic identity that expands products of skew elements in terms of the coproduct and antipode.

Résumé. Nous produisons quelques règles dissymétrique de Pieri pour les fonctions Hall-Littlewood au sens de Assaf et McNamara (FPSAC, 2010). Les premières deux règles ont été conjecturée par le premier auteur (FPSAC, 2011). Les principaux ingrédients dans les preuves sont une identité $q$-binomiale pour les partitions dissymétrique qui sont bandes horizontales et une identité de Hopf qui exprime les produits d'éléments dissymétrique en termes du coproduit et de l'antipode.
\end{abstract}

Keywords: Pieri Rules, Hall-Littlewood functions

Let $\Lambda[t]$ denote the ring of symmetric functions over $\mathbb{Q}(t)$, and let $\left\{s_{\lambda}\right\}$ and $\left\{P_{\lambda}(t)\right\}$ denote its bases of Schur functions and Hall-Littlewood functions, respectively, indexed by partitions $\lambda$. The Schur functions lead a rich life-making appearances in combinatorics, representation theory and Schubert calculus, among other places. See [Ful97, Mac95] for details. The Hall-Littlewood functions are nearly as ubiquitous (having as a salient feature that $P_{\lambda}(t) \rightarrow s_{\lambda}$ under the specialization $t \rightarrow 0$ ). See [LLT97] and the references therein for their place in the literature.

A classical problem is to determine cancellation-free formulas for multiplication in these bases,

$$
s_{\lambda} s_{\mu}=\sum_{\nu} c_{\lambda, \mu}^{\nu} s_{\nu} \quad \text { and } \quad P_{\lambda} P_{\mu}=\sum_{\nu} f_{\lambda, \mu}^{\nu}(t) P_{\nu}
$$

The first problem was only given a complete solution in the latter half of the 20th century, while the second problem remains open. Special cases of the problem, known as Pieri rules, have been understood for quite a bit longer. The Pieri rules for Schur functions [Mac95, Ch. I, (5.16) and (5.17)] take the form

$$
s_{\lambda} s_{1^{r}}=s_{\lambda} e_{r}=\sum_{\lambda^{+}} s_{\lambda^{+}},
$$

\footnotetext{
${ }^{\dagger}$ M. Konvalinka supported in part by Research Programs P1-0294 and P1-0297 of the Slovenian Research Agency

$\ddagger$ A. Lauve supported in part by NSA grant \#H98230-10-1-0362. 
with the sum over partitions $\lambda^{+}$for which $\lambda^{+} / \lambda$ is a vertical strip of size $r$, and

$$
s_{\lambda} s_{r}=\sum_{\lambda^{+}} s_{\lambda^{+}},
$$

with the sum over partitions $\lambda^{+}$for which $\lambda^{+} / \lambda$ is a horizontal strip of size $r$. (See Section 1 for the definitions of vertical- and horizontal strip.) The Pieri rules for Hall-Littlewood functions [Mac95, Ch. III, (3.2) and (5.7)] state that

$$
P_{\lambda} P_{1^{r}}=P_{\lambda} e_{r}=\sum_{\left|\lambda^{+} / \lambda\right|=r} \mathrm{vs}_{\lambda^{+} / \lambda}(t) P_{\lambda^{+}}
$$

and

$$
P_{\lambda} q_{r}=\sum_{\left|\lambda^{+} / \lambda\right|=r} \mathrm{hs}_{\lambda^{+} / \lambda}(t) P_{\lambda^{+}},
$$

with the sums again running over vertical strips and horizontal strips, respectively. Here $q_{r}$ denotes $(1-t) P_{r}$ for $r>0$ with $q_{0}=P_{0}=1$, and $\mathrm{vs}_{\lambda / \mu}(t), \mathrm{hs}_{\lambda / \mu}(t)$ are certain polynomials in $t$. (See Section 1 for their definitions, as well as those of $\operatorname{sk}_{\lambda / \mu}(t)$ and $\mathrm{br}_{\lambda / \mu}(t)$ appearing below.)

Our first result is a Pieri-type mixture of the two bases.

Theorem 1 For a partition $\lambda$ and $r \geq 0$, we have

$$
P_{\lambda} s_{r}=\sum_{\lambda^{+}} \operatorname{sk}_{\lambda^{+} / \lambda}(t) P_{\lambda^{+}}
$$

with the sum over partitions $\lambda^{+} \supseteq \lambda$ for which $\left|\lambda^{+} / \lambda\right|=r$.

The main focus of this article is on the generalizations of Hall-Littlewood functions to skew shapes $\lambda / \mu$. We introduce the question via the recent answer for skew Schur functions $s_{\lambda / \mu}$. In [AM11], Assaf and McNamara give a skew Pieri rule for Schur functions. They prove the following generalization of (2):

$$
s_{\lambda / \mu} s_{r}=\sum_{\lambda^{+}, \mu^{-}}(-1)^{\mid \mu / \mu^{-}} s_{\lambda^{+} / \mu^{-}},
$$

with the sum over pairs $\left(\lambda^{+}, \mu^{-}\right)$of partitions such that $\lambda^{+} / \lambda$ is a horizontal strip, $\mu / \mu^{-}$is a vertical strip and $\left|\lambda^{+} / \lambda\right|+\left|\mu / \mu^{-}\right|=r$. This elegant gluing-together of an $s_{r}$-type Pieri rule for the outer rim of $\lambda / \mu$ with an $e_{r}$-type Pieri rule for the inner rim of $\lambda / \mu$ demanded further exploration.

In [AM11], Lam, Sottile and the second author [LLS11] found a Hopf algebraic explanation for [6] that readily extended to many other settings. (For example, a skew Pieri rule for $k$-Schur functions was given.) Within the setting of Schur functions, it provided an easy extension of (6) to products of arbitrary skew Schur functions-a formula first conjectured by Assaf and McNamara in [AM11]. The results of this paper use the same Hopf machinery. We reprise most of the details and background in Section2.

Around the same time, the first author [Kon] was motivated to give a skew Murnaghan-Nakayama rule in the spirit of [AM11]. Along the way, he gives a bijective proof of the conjugate form of (6) (only proven in [AM11] using the automorphism $\omega$ ) and a quantum skew Murnaghan-Nakayama rule:

$$
s_{\lambda / \mu} q_{r}=\sum_{\lambda^{+}, \mu^{-}}(-1)^{\left|\mu / \mu^{-}\right|} \operatorname{br}_{\lambda^{+} / \lambda}(t) \operatorname{br}_{\left(\mu / \mu^{-}\right)^{c}}(t) s_{\lambda^{+} / \mu^{-}},
$$


with the sum over pairs $\left(\lambda^{+}, \mu^{-}\right)$of partitions such that $\lambda^{+} / \lambda$ and $\mu / \mu^{-}$are broken ribbons and $\left|\lambda^{+} / \lambda\right|+$ $\left|\mu / \mu^{-}\right|=r$. Note that since $P_{r}(0)=s_{r}$, we recover the skew Pieri rule for $t=0$. Also, since $P_{r}(1)=p_{r}$, we recover a skew Murnaghan-Nakayama rule if we divide the formula by $1-t$ and let $t \rightarrow 1$. This formula, like that in Theorem 1. may be viewed as a link between the two theories of Schur and HallLittlewood functions. One might ask for other examples of mixing, e.g., swapping the rolls of Schur and Hall-Littlewood functions in (7). Two such examples were found (conjecturally) in [Kon]. Their proofs, and a generalization of (6) to the Hall-Littlewood setting, are the main results of this paper.

Theorem 2 For partitions $\lambda, \mu, \mu \subseteq \lambda$, and $r \geq 0$, we have

$$
P_{\lambda / \mu} s_{1^{r}}=P_{\lambda / \mu} e_{r}=P_{\lambda / \mu} P_{1^{r}}=\sum_{\lambda^{+}, \mu^{-}}(-1)^{\left|\mu / \mu^{-}\right|} \mathrm{vs}_{\lambda^{+} / \lambda}(t) \mathrm{sk}_{\mu / \mu^{-}}(t) P_{\lambda^{+} / \mu^{-}}
$$

where the sum on the right is over all $\lambda^{+} \supseteq \lambda, \mu^{-} \subseteq \mu$ such that $\left|\lambda^{+} / \lambda\right|+\left|\mu / \mu^{-}\right|=r$.

Theorem 3 For partitions $\lambda, \mu, \mu \subseteq \lambda$, and $r \geq 0$, we have

$$
P_{\lambda / \mu} s_{r}=\sum_{\lambda^{+}, \mu^{-}}(-1)^{\left|\mu / \mu^{-}\right|} \operatorname{sk}_{\lambda^{+} / \lambda}(t) \mathrm{vs}_{\mu / \mu^{-}}(t) P_{\lambda^{+} / \mu^{-}}
$$

where the sum on the right is over all $\lambda^{+} \supseteq \lambda, \mu^{-} \subseteq \mu$ such that $\left|\lambda^{+} / \lambda\right|+\left|\mu / \mu^{-}\right|=r$.

Note that putting $\mu=\emptyset$ above recovers Theorem 1 .

Theorem 4 For partitions $\lambda, \mu, \mu \subseteq \lambda$, and $r \geq 0$, we have

$$
P_{\lambda / \mu} q_{r}=\sum_{\lambda^{+}, \mu^{-}, \tau}(-1)^{\left|\mu / \mu^{-}\right|}(-t)^{\left|\tau / \mu^{-}\right|} \mathrm{hs}_{\lambda^{+} / \lambda}(t) \mathrm{vs}_{\mu / \tau}(t) \mathrm{sk}_{\tau / \mu^{-}}(t) P_{\lambda^{+} / \mu^{-}},
$$

where the sum on the right is over all $\lambda^{+} \supseteq \lambda, \mu^{-} \subseteq \tau \subseteq \mu$ such that $\left|\lambda^{+} / \lambda\right|+\left|\mu / \mu^{-}\right|=r$.

This paper is organized as follows. In Section 1 , we prove some polynomial identities involving hs, vs and sk, prove Theorem 1, and find $\omega\left(q_{r}\right)$. In Section 2, we introduce our main tool, Hopf algebras. We conclude in Section 3 with the proofs of our main theorems.

\section{Combinatorial Preliminaries}

\subsection{Notation and a key lemma}

The conjugate partition of $\lambda$ is denoted $\lambda^{c}$. We write $m_{i}(\lambda)$ for the number of parts of $\lambda$ equal to $i$. For a partition $\lambda$, define $n(\lambda)=\sum_{i}(i-1) \lambda_{i}=\sum_{i}\left(\begin{array}{c}\lambda_{i}^{c} \\ 2\end{array}\right)$. The $q$-binomial coefficient is defined by

$$
\left[\begin{array}{l}
a \\
b
\end{array}\right]_{q}=\frac{\left(1-q^{a}\right)\left(1-q^{a-1}\right) \cdots\left(1-q^{a-b+1}\right)}{\left(1-q^{b}\right)\left(1-q^{b-1}\right) \cdots(1-q)}
$$

and is a polynomial in $q$ that gives $\left(\begin{array}{l}a \\ b\end{array}\right)$ when $q=1$.

We say that $\lambda / \mu$ is a horizontal strip (respectively vertical strip) if $[\lambda / \mu]$ contains no $2 \times 1$ (respectively $1 \times 2$ ) block, equivalently, if $\lambda_{i}^{c} \leq \mu_{i}^{c}+1$ (respectively $\lambda_{i} \leq \mu_{i}+1$ ) for all $i$. We say that $\lambda / \mu$ is a ribbon 
if $[\lambda / \mu]$ is connected and if it contains no $2 \times 2$ block, and that $\lambda / \mu$ is a broken ribbon if $[\lambda / \mu]$ contains no $2 \times 2$ block, equivalently, if $\lambda_{i} \leq \mu_{i-1}+1$ for $i \geq 2$. The Young diagram of a broken ribbon is a disjoint union of $\operatorname{rib}(\lambda / \mu)$ number of ribbons. The height $\operatorname{ht}(\lambda / \mu)$ (respectively width $\mathrm{wt}(\lambda / \mu)$ ) of a ribbon is the number of non-empty rows (respectively columns) of $[\lambda / \mu]$, minus 1 . The height (respectively width) of a broken ribbon is the sum of heights (respectively widths) of the components.

Let us define some polynomials. For a horizontal strip $\lambda / \mu$, define

$$
\mathrm{hs}_{\lambda / \mu}(t)=\prod_{\substack{\lambda_{j}^{c}=\mu_{j}^{c}+1 \\ \lambda_{j+1}^{c}=\mu_{j+1}^{c}}}\left(1-t^{m_{j}(\lambda)}\right) .
$$

If $\lambda / \mu$ is not a horizontal strip, define $\mathrm{hs}_{\lambda / \mu}(t)=0$. For a vertical strip $\lambda / \mu$, define

$$
\operatorname{vs}_{\lambda / \mu}(t)=\prod_{j \geq 1}\left[\begin{array}{c}
\lambda_{j}^{c}-\lambda_{j+1}^{c} \\
\lambda_{j}^{c}-\mu_{j}^{c}
\end{array}\right]_{t}
$$

If $\lambda / \mu$ is not a vertical strip, define $\operatorname{vs}_{\lambda / \mu}(t)=0$. For a broken ribbon $\lambda / \mu$, define

$$
\mathrm{br}_{\lambda / \mu}(t)=(-t)^{\mathrm{ht}(\lambda / \mu)}(1-t)^{\mathrm{rib}(\lambda / \mu)}
$$

If $\lambda / \mu$ is not a broken ribbon, define $\operatorname{br}_{\lambda / \mu}(t)=0$. For any skew shape $\lambda / \mu$, define

$$
\operatorname{sk}_{\lambda / \mu}(t)=t^{\sum_{j}\left(\begin{array}{c}
\lambda_{j}^{c}-\mu_{j}^{c} \\
2
\end{array}\right)} \prod_{j \geq 1}\left[\begin{array}{c}
\lambda_{j}^{c}-\mu_{j+1}^{c} \\
m_{j}(\mu)
\end{array}\right]_{t} .
$$

Lemma 5 For fixed $\lambda, \mu, \mu \subseteq \lambda$, we have

$$
\sum_{\nu}(-t)^{|\lambda / \nu|} \operatorname{vs}_{\lambda / \nu}(t) \operatorname{sk}_{\nu / \mu}(t)=\mathrm{hs}_{\lambda / \mu}(t)
$$

with the sum over all $\nu, \mu \subseteq \nu \subseteq \lambda$, for which $\lambda / \nu$ is a vertical strip.

Proof: Let $a_{j}=\lambda_{j}^{c}-\max \left(\mu_{j}^{c}, \lambda_{j+1}^{c}\right) \geq 0$. A partition $\nu, \mu \subseteq \nu \subseteq \lambda$, for which $\lambda / \nu$ is a vertical strip is obtained by choosing $k_{j}, 0 \leq k_{j} \leq a_{j}$, and removing $k_{j}$ bottom cells of column $j$ in $\lambda$. See Figure 1 for the example $\lambda=98886666444$ and $\mu=77666633331$, where $a_{4}=3, a_{6}=2, a_{8}=3, a_{9}=1$ and $a_{i}=0$ for all other $i$.

We have $|\lambda / \nu|=\sum_{j} k_{j}, \nu_{j}^{c}=\lambda_{j}^{c}-k_{j}$. We make all such choices independently, which means that

$$
\begin{gathered}
\sum_{\nu}(-t)^{|\lambda / \nu|} \operatorname{sk}_{\nu / \mu}(t) \operatorname{vs}_{\lambda / \nu}(t)=\sum_{k_{1}, k_{2}, \ldots}(-t)^{\sum_{j} k_{j}} t^{\sum_{j}\left(\begin{array}{c}
\nu_{j}^{c}-\mu_{j}^{c} \\
2
\end{array}\right)} \prod_{j}\left[\begin{array}{c}
\nu_{j}^{c}-\mu_{j+1}^{c} \\
m_{j}(\mu)
\end{array}\right]_{t} \prod_{j}\left[\begin{array}{c}
\lambda_{j}^{c}-\lambda_{j+1}^{c} \\
\lambda_{j}^{c}-\nu_{j}^{c}
\end{array}\right]_{t} \\
=\prod_{j} \sum_{k_{j}=0}^{a_{j}}(-t)^{k_{j}} t^{\left(\begin{array}{c}
\lambda_{j}^{c}-\mu_{j}^{c}-k_{j} \\
2
\end{array}\right)}\left[\begin{array}{c}
\lambda_{j}^{c}-k_{j}-\mu_{j+1}^{c} \\
m_{j}(\mu)
\end{array}\right]_{t}\left[\begin{array}{c}
m_{j}(\lambda) \\
k_{j}
\end{array}\right]_{t}
\end{gathered}
$$




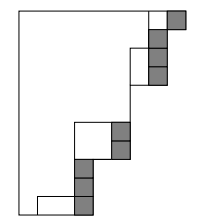

Fig. 1: A partition $\nu(\mu \subseteq \nu \subseteq \lambda)$ for which $\lambda / \nu$ is a vertical strip within $\lambda / \mu$ is built from $\lambda$ by removing some number of the shaded cells of $\lambda$.

The rest of the proof consists of an involved case-by-case analysis which repeatedly uses the $q$-binomial theorem. Specifically, first we show that if $\lambda / \mu$ is a horizontal strip, then the term in the product 8 corresponding to $j$ is $1-t^{m_{j}(\lambda)}$ if $\lambda_{j}^{c}=\mu_{j}^{c}+1, \lambda_{j+1}^{c}=\mu_{j+1}^{c}$, and 1 otherwise. Next, we show that if $\lambda / \mu$ is not a horizontal strip and $j$ is the largest index for which $\lambda_{j}^{c}-\mu_{j}^{c} \geq 2$, then the term in the product (8) corresponding to $j$ is 0 .

\subsection{Elementary Hall-Littlewood identities}

We give two applications of Lemma 5, then prove some elementary properties of Hall-Littlewood functions that will be useful in Section 3 . The first application is a formula for the product of a Hall-Littlewood polynomial with the Schur function $s_{r}$.

Proof of Theorem 1: We induct on $r$. For $r=0$, there is nothing to prove. For $r>0$, we use the formula

$$
q_{r}=\sum_{k=0}^{r}(-t)^{k} s_{r-k} e_{k},
$$

which is easy to prove using, say, [Sta99, Exercise 7.11]) and the conjugate Pieri rule. For $\left|\lambda^{+} / \lambda\right|=r$, the coefficient of $P_{\lambda^{+}}$in

$$
P_{\lambda} s_{r}=P_{\lambda}\left(q_{r}-\sum_{k=1}^{r}(-t)^{k} s_{r-k} e_{k}\right)
$$

is (by (3), (4) and induction) equal to

$$
\mathrm{hs}_{\lambda+/ \lambda}(t)-\sum(-t)^{\left|\lambda^{+} / \nu\right|} \operatorname{sk}_{\nu / \lambda}(t) \operatorname{vs}_{\lambda^{+} / \nu}(t),
$$

with the sum over all $\nu, \lambda \subseteq \nu \subseteq \lambda^{+}$, for which $\lambda^{+} / \nu$ is a vertical strip of size at least 1 . By Lemma 5 , this is equal to $\operatorname{sk}_{\lambda^{+} / \lambda}(t)$.

Recall that $f_{\sigma, \tau}^{\lambda}(t)$ is the (polynomial) coefficient of $P_{\lambda}$ in $P_{\sigma} P_{\tau}$.

Corollary 6 The structure constants $f_{\mu, \tau}^{\lambda}(t)$ satisfy $\sum_{\tau} t^{n(\tau)} f_{\mu, \tau}^{\lambda}(t)=\operatorname{sk}_{\lambda / \mu}(t)$.

Proof: This follows from $s_{r}=\sum_{\tau \vdash r} t^{n(\tau)} P_{\tau}$, which is (2) in [Mac95, page 219] and also Theorem 1 for $\lambda=\emptyset$.

The second application of Lemma 5 is a generalization of [Mac95, §III.3, Example 1]. 
Theorem 7 For every $\lambda, \mu$, we have

$$
\sum_{\nu} \operatorname{sk}_{\nu / \mu}(t) \operatorname{vs}_{\lambda / \nu}(t) y^{|\lambda / \nu|}=\sum_{\sigma} t^{n(\sigma)-\left(\begin{array}{c}
\ell(\sigma) \\
2
\end{array}\right)} f_{\sigma \mu}^{\lambda}(t) \prod_{j=1}^{\ell(\sigma)}\left(y+t^{j-1}\right) .
$$

Equivalently, for all m,

$$
\sum_{\nu:|\lambda / \nu|=m} \operatorname{sk}_{\nu / \mu}(t) \operatorname{vs}_{\lambda / \nu}(t)=\sum_{\sigma} t^{n(\sigma)-\left(\begin{array}{c}
m \\
2
\end{array}\right)} f_{\sigma \mu}^{\lambda}(t)\left[\begin{array}{c}
\ell(\sigma) \\
m
\end{array}\right]_{t^{-1}} .
$$

Proof: Let us evaluate $P_{\mu} s_{r}\left(\sum_{m} e_{m} y^{m}\right)$ in two different ways. On the one hand,

$$
P_{\mu} s_{r}\left(\sum_{m} e_{m} y^{m}\right)=\left(\sum_{\nu} \mathrm{sk}_{\nu / \mu}(t) P_{\nu}\right)\left(\sum_{m} e_{m} y^{m}\right)=\sum_{\nu, \lambda} \operatorname{sk}_{\nu / \mu}(t) \operatorname{vs}_{\lambda / \nu}(t) P_{\lambda} y^{|\lambda / \nu|} .
$$

On the other hand, using Example 1 on page 218 of [Mac95],

$$
P_{\mu} s_{r}\left(\sum_{m} e_{m} y^{m}\right)=P_{\mu} \sum_{\sigma} t^{n(\sigma)} P_{\sigma} \prod_{j=1}^{\ell(\sigma)}\left(1+t^{1-j} y\right)=\sum_{\sigma, \lambda} t^{n(\sigma)-(\stackrel{\ell(\sigma)}{2})} f_{\sigma \mu}^{\lambda}(t) P_{\lambda} \prod_{j=1}^{\ell(\sigma)}\left(y+t^{j-1}\right) .
$$

Now (10) follows by taking the coefficient of $P_{\lambda}$ in both expressions. For (11), we use the $q$-binomial theorem and

$$
\left[\begin{array}{l}
n \\
k
\end{array}\right]_{t^{-1}}=t^{\left(\begin{array}{c}
k \\
2
\end{array}\right)+\left(\begin{array}{c}
n-k \\
2
\end{array}\right)-\left(\begin{array}{c}
n \\
2
\end{array}\right)}\left[\begin{array}{l}
n \\
k
\end{array}\right]_{t}
$$

Remark 8 The theorem is indeed a generalization of [Mac95, §III.3, Example 1]. For $\mu=\emptyset, \mathrm{sk}_{\nu / \mu}(t)=$ $t^{n(\nu)}$, and the right-hand side of (11) is non-zero only for $\sigma=\lambda$, so the last equation on page 218 (loc. cit.) follows. It also generalizes Lemma 5 for $y=-t$, the right-hand side of (10) is non-zero if and only if $\ell(\sigma)=1$, and is therefore equal to $\mathrm{hs}_{\lambda / \mu}(t)$.

We finish the section with two more lemmas.

Lemma 9 Given $r>k \geq 0$, we have

$$
s_{r-k, 1^{k}}=\sum_{\lambda: \ell(\lambda) \geq k+1} t\left(\begin{array}{c}
\ell(\lambda)-k \\
2
\end{array}\right)+\sum_{i=2}^{\lambda_{1}}\left(\begin{array}{c}
\lambda_{i}^{c} \\
2
\end{array}\right)\left[\begin{array}{c}
\ell(\lambda)-1 \\
k
\end{array}\right]_{t} P_{\lambda} .
$$

Proof: The lemma follows from the formula due to Lascoux and Schützenberger, see, e.g., [Mac95, Ch. III, (6.5)]. In that terminology, we have to evaluate $K_{\left(r-k, 1^{k}\right), \lambda}(t)$. We choose a semistandard Young tableau $T$ of shape $\left(r-k, 1^{k}\right)$ and type $\lambda=\left(\lambda_{1}, \ldots, \lambda_{\ell}\right)$. Clearly, such tableaux are in one-to-one correspondence with $k$-subsets of the set $\{2, \ldots, \ell\}$. For such a subset $S$, write $s$ for the word with the elements of $S$ in increasing order, and write $\bar{s}$ for the word with the elements of $\{2, \ldots, \ell\} \backslash S$ in decreasing 
order. The reading word of the tableau corresponding to $S$ is $\ell^{\lambda_{\ell}-1} \cdots 3^{\lambda_{3}-1} 2^{\lambda_{2}-1} 1^{\lambda_{1}} s$. The subwords $w_{2}, w_{3}, \ldots$ are all strictly decreasing, and $w_{1}=\bar{s} 1 s$. The charges of $w_{2}, w_{3}, \ldots$ are $\left(\begin{array}{c}\lambda_{2}^{c} \\ 2\end{array}\right),\left(\begin{array}{c}\lambda_{3}^{c} \\ 2\end{array}\right), \ldots$, while the charge of $w_{1}$ is $\sum_{i \notin S}(\ell-i+1)$. The formula

$$
\sum_{S \subseteq\{2, \ldots, \ell+1\},|S|=k} t^{\sum_{i \notin S}(\ell-i+1)}=t^{\left(\begin{array}{l}
\ell-k \\
2
\end{array}\right)}\left[\begin{array}{c}
\ell-1 \\
k
\end{array}\right]_{t}
$$

can be proved by induction.

Lemma 10 Let $\omega$ be the fundamental involution on $\Lambda[t]$ defined by $\omega\left(s_{\lambda}\right)=s_{\lambda^{c}}$. We have

$$
\omega\left(q_{r}\right)=(-1)^{r} \sum_{\lambda \vdash r} c_{\lambda}(t) P_{\lambda},
$$

where

$$
c_{\lambda}(t)=t^{\sum_{i=2}^{\lambda_{1}}\left(\begin{array}{c}
\lambda_{i}^{c}+1 \\
2
\end{array}\right)} \prod_{i=1}^{\ell(\lambda)}\left(-1+t^{i}\right)
$$

Proof: We have

$$
\begin{aligned}
& \omega\left(P_{r}\right)=\omega\left(\sum_{k=0}^{r-1}(-t)^{r-k-1} s_{k+1,1^{r-k-1}}\right)=\sum_{k=0}^{r-1}(-t)^{r-k-1} s_{r-k, 1^{k}}=
\end{aligned}
$$

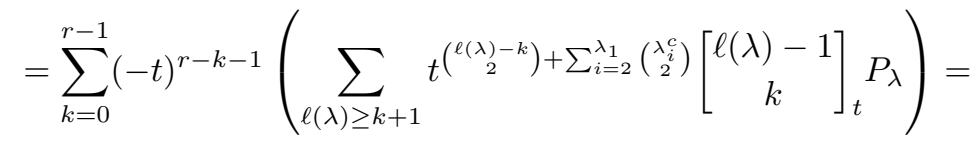

$$
\begin{aligned}
& =\sum_{\lambda \vdash r}\left(\sum_{k=0}^{\ell(\lambda)-1}(-t)^{r-k-1} t^{\left(\begin{array}{l}
\ell(\lambda)-k \\
2
\end{array}\right)+\sum_{i=2}^{\lambda_{1}}\left(\begin{array}{c}
\lambda_{i}^{c} \\
2
\end{array}\right)}\left[\begin{array}{c}
\ell(\lambda)-1 \\
k
\end{array}\right]_{t}\right) P_{\lambda} .
\end{aligned}
$$

Now by the $q$-binomial theorem,

$$
\left.\prod_{i=2}^{\ell(\lambda)}\left(-1+t^{i}\right)=t^{2(\ell(\lambda)-1)} \prod_{i=0}^{\ell(\lambda)-2}\left(-1 / t^{2}+t^{i}\right)=t^{2(\ell(\lambda)-1)} \sum_{k=0}^{\ell(\lambda)-1} t^{(\ell(\lambda)-1-k}\right)\left[\begin{array}{c}
\ell(\lambda)-1 \\
k
\end{array}\right]_{t}\left(-\frac{1}{t^{2}}\right)^{k}
$$

and simple calculations show that the coefficient of $P_{\lambda}$ in $\omega\left(q_{r}\right)=(1-t) \omega\left(P_{r}\right)$ is indeed $(-1)^{r} c_{\lambda}(t)$.

\section{Hopf Perspective on Skew Elements}

Recall that $\Lambda[t]$ has another important basis $\left\{Q_{\lambda}\right\}$, defined by $Q_{\lambda}=b_{\lambda}(t) P_{\lambda}$, where $b_{\lambda}(t)=\prod_{i \geq 1}(1-$ $t)\left(1-t^{2}\right) \cdots\left(1-t^{m_{i}(\lambda)}\right)$. The (extended) Hall scalar product on $\Lambda[t]$ is defined by either of the equivalent conditions

$$
\left\langle P_{\lambda}, Q_{\mu}\right\rangle=\delta_{\lambda \mu} \quad \text { or } \quad\left\langle p_{\lambda}, p_{\mu}\right\rangle=z_{\mu}(t) \delta_{\lambda \mu},
$$


where $z_{\mu}(t):=z_{\mu} \cdot \prod_{j=1}^{r}\left(1-t^{\mu_{j}}\right)^{-1}=\prod_{i=1}^{k}\left(i^{a_{i}} a_{i} !\right) \prod_{j=1}^{r}\left(1-t^{\mu_{j}}\right)^{-1}$ for $\mu=\left(\mu_{1}, \mu_{2}, \ldots, \mu_{r}\right)=$ $\left\langle 1^{a_{1}}, 2^{a_{2}}, \cdots, k^{a_{k}}\right\rangle$. See [Mac95, §III.4]. The skew Hall-Littlewood function $P_{\lambda / \mu}$ is defined [Mac95. Ch. III, $\left.\left(5.1^{\prime}\right)\right]$ as the unique function satisfying

$$
\left\langle P_{\lambda / \mu}, Q_{\nu}\right\rangle=\left\langle P_{\lambda}, Q_{\nu} Q_{\mu}\right\rangle
$$

for all $Q_{\nu} \in \Lambda[t]$. (Likewise for $Q_{\lambda / \mu}$.) If we choose to read $P_{\lambda / \mu}$ as, " $Q_{\mu}$ skews $P_{\lambda}$," then we allow ourselves access to the machinery of Hopf algebra actions on their duals. We introduce the basics in Subsection 2.1 and return to $\Lambda[t]$ and Hall-Littlewood functions in Subsection 2.2 .

\subsection{Hopf preliminaries}

Let $H=\bigoplus_{n} H_{n}$ be a graded algebra over a field $\mathbb{k}$. Recall that $H$ is a Hopf algebra if there are algebra maps $\Delta: H \rightarrow H \otimes H$ and $\varepsilon: H \rightarrow \mathbb{k}$, and an algebra antimorphism $S: H \rightarrow H$, called the coproduct, counit and antipode, respectively, satisfying some additional compatibility conditions. See [Mon93]. We use Sweedler's notation for the coproduct, denoting $\Delta(h)$ by $\sum_{(h)} h^{\prime} \otimes h^{\prime \prime}$ for $h \in H$.

Let $H^{*}=\bigoplus_{n} H_{n}^{*}$ denote the graded dual of $H$. If each $H_{n}$ is finite dimensional, then the pairing $\langle\cdot, \cdot\rangle: H \otimes H^{*} \rightarrow \mathbb{k}$ defined by $\langle h, a\rangle=a(h)$ is nondegenerate. This pairing naturally endows $H^{*}$ with a Hopf algebra structure, with product and coproduct uniquely determined by the formulas:

$$
\langle h, a \cdot b\rangle:=\langle\Delta(h), a \otimes b\rangle \quad \text { and } \quad\langle g \otimes h, \Delta(a\rangle):=\langle g \cdot h, a\rangle
$$

for all homogeneous $g, h \in H$ and $a, b \in H^{*}$. (Extend to all of $H^{*}$ by linearity, insisting that $\left\langle H_{n}, H_{m}^{*}\right\rangle=$ 0 for $n \neq m$.)

We recall some standard actions ("- ") of $H$ and $H^{*}$ on each other. Given $h \in H$ and $a \in H^{*}$, put

$$
a \rightarrow h:=\sum_{(h)}\left\langle h^{\prime \prime}, a\right\rangle h^{\prime} \quad \text { and } \quad h \rightarrow a:=\sum_{(a)}\left\langle h, a^{\prime \prime}\right\rangle a^{\prime} .
$$

Equivalently, $\langle g, h \rightarrow a\rangle=\langle g \cdot h, a\rangle$ and $\langle a \rightarrow h, b\rangle=\langle h, b \cdot a\rangle$. We call these skew elements (in $H$ and $H^{*}$, respectively) to keep the nomenclature consistent with that in symmetric function theory.

Our skew Pieri rules (Theorems 2,3 and 4) come from an elementary formula relating products of elements $h$ and skew elements $a \rightarrow g$ in a Hopf algebra $H$ :

$$
(a \rightarrow g) \cdot h=\sum\left(S\left(h^{\prime \prime}\right) \rightarrow a\right) \rightarrow\left(g \cdot h^{\prime}\right)
$$

See $(*)$ in the proof of [Mon93, Lemma 2.1.4] or [LLS11, Lemma 1]. Before turning to the proofs of these theorems, we first recall the Hopf structure of $\Lambda[t]$.

\subsection{The Hall-Littlewood setting}

The ring $\Lambda[t]$ is generated by the one-part power sum symmetric functions $p_{r}(r>0)$, so the definitions

$$
\Delta\left(p_{r}\right):=1 \otimes p_{r}+p_{r} \otimes 1, \quad \varepsilon\left(p_{r}\right):=0, \quad \text { and } \quad S\left(p_{r}\right):=-p_{r}
$$

completely determine the Hopf structure of $\Lambda[t]$. 
Proposition 11 For $r>0$ and $c_{\lambda}$ given by Lemma 10

$$
\begin{aligned}
& \Delta\left(e_{r}\right)=\sum_{k=0}^{r} e_{k} \otimes e_{r-k} \quad \Delta\left(s_{r}\right)=\sum_{k=0}^{r} s_{k} \otimes s_{r-k} \quad \Delta\left(q_{r}\right)=\sum_{k=0}^{r} q_{k} \otimes q_{r-k} \\
& S\left(e_{r}\right)=(-1)^{r} s_{r} \quad S\left(s_{r}\right)=(-1)^{r} e_{r} \quad S\left(q_{r}\right)=\sum_{\lambda \vdash r} c_{\lambda} P_{\lambda} .
\end{aligned}
$$

Proof: Equalities for $e_{r}$ and $s_{r}$ are elementary consequences of (15) and may be found in [Mac95, $\S \mathrm{I} .5$, Example 25]. The coproduct formula for $q_{r}$ is (2) in [Mac95, §III.5, Example 8]. The antipode formula for $q_{r}$ is identical to Lemma 10 , as the fundamental morphism $\omega$ and the antipode $S$ are related by $S(h)=$ $(-1)^{r} \omega(h)$ on homogeneous elements $h$ of degree $r$.

It happens that $\Lambda[t]$ is a self-dual Hopf algebra. This may be deduced from [Mac95, §III.5, Example 8] and is easy to see on the power sum basis. (Write $p_{\lambda}^{*}$ for $z_{\lambda}(t)^{-1} p_{\lambda}$ and use (15) to check that $\left\langle p_{\lambda}, p_{\mu}^{*} \cdot p_{\nu}^{*}\right\rangle=\left\langle\Delta\left(p_{\lambda}\right), p_{\mu}^{*} \otimes p_{\nu}^{*}\right\rangle$ and $\left\langle p_{\mu} \otimes p_{\nu}, \Delta\left(p_{\lambda}^{*}\right)\right\rangle=\left\langle p_{\mu} \cdot p_{\nu}, p_{\lambda}^{*}\right\rangle$ for all partitions $\lambda, \mu$ and $\nu$.)

After (12), (13) and self-duality, we see that $P_{\lambda / \mu}=Q_{\mu} \rightarrow P_{\lambda}$, and similarly, $Q_{\lambda / \mu}=P_{\mu} \rightarrow Q_{\lambda}$.

\section{Proofs of the main theorems}

We specialize (14) to Hall-Littlewood polynomials, putting $a \rightarrow g=P_{\lambda / \mu}$.

Proof of Theorem 2; Taking $h=e_{r}$ in (14), we get

$$
\begin{aligned}
P_{\lambda / \mu} \cdot e_{r}= & \left(Q_{\mu} \rightarrow P_{\lambda}\right) \cdot e_{r}=\sum_{\left(e_{r}\right)}\left(S\left(e_{r}^{\prime \prime}\right) \rightarrow Q_{\mu}\right) \rightarrow\left(P_{\lambda} \cdot e_{r}{ }^{\prime}\right) \\
= & \sum_{k=0}^{r}\left(S\left(e_{k}\right) \rightarrow Q_{\mu}\right) \rightarrow\left(P_{\lambda} \cdot e_{r-k}\right) \\
= & \sum_{k=0}^{r}(-1)^{k}\left(s_{k} \rightarrow Q_{\mu}\right) \rightarrow\left(P_{\lambda} \cdot e_{r-k}\right) \\
= & \sum_{k=0}^{r}(-1)^{k}\left(\sum_{\tau} t^{n(\tau)} Q_{\mu / \tau}\right) \rightarrow\left(P_{\lambda} \cdot e_{r-k}\right) \\
= & \sum_{k=0}^{r}(-1)^{k}\left(\sum_{\left|\mu / \mu^{-}\right|=k}\left(\sum_{\tau} t^{n(\tau)} f_{\mu^{-}, \tau}^{\mu}(t)\right) Q_{\mu^{-}}\right) \rightarrow\left(\sum_{\left|\lambda^{+} / \lambda\right|=r-k} \mathrm{vs}_{\lambda^{+} / \lambda}(t) P_{\lambda^{+}}\right) \\
= & \sum_{\lambda^{+}, \mu^{-}}(-1)^{\left|\mu / \mu^{-}\right|} \operatorname{sk}_{\mu / \mu^{-}}(t) \operatorname{vs}_{\lambda^{+} / \lambda}(t) P_{\lambda^{+} / \mu^{-}} \cdot
\end{aligned}
$$

For (17) and 18), we used Proposition 11. For (19), we used $s_{k} \rightarrow Q_{\mu}=\left(\sum_{\tau \vdash k} t^{n(\tau)} P_{\tau}\right) \rightarrow Q_{\mu}=$ $\sum_{\tau \vdash k} t^{n(\tau)} Q_{\mu / \tau}$. We use (3) and (12) to pass from (19) to (20). Explicitly, the coefficient of $Q_{\mu^{-}}$in the expansion of $Q_{\mu / \tau}$ is equal to the coefficient of $P_{\mu}$ in $P_{\mu^{-}} P_{\tau}$. Finally, (21) follows from Corollary 6. 
Proof of Theorem 3; Taking $h=s_{r}$ in (14), we get

$$
\begin{aligned}
P_{\lambda / \mu} \cdot s_{r}= & \left(Q_{\mu} \rightarrow P_{\lambda}\right) \cdot s_{r}=\sum_{\left(s_{r}\right)}\left(S\left(s_{r}{ }^{\prime \prime}\right) \rightarrow Q_{\mu}\right) \rightarrow\left(P_{\lambda} \cdot s_{r}{ }^{\prime}\right) \\
& =\sum_{k=0}^{r}\left(S\left(s_{k}\right) \rightarrow Q_{\mu}\right) \rightarrow\left(P_{\lambda} \cdot s_{r-k}\right) \\
& =\sum_{k=0}^{r}(-1)^{k}\left(e_{k} \rightarrow Q_{\mu}\right) \rightarrow\left(P_{\lambda} \cdot s_{r-k}\right) \\
& =\sum_{k=0}^{r}(-1)^{k} Q_{\mu / 1^{k}} \rightarrow\left(P_{\lambda} \cdot s_{r-k}\right) \\
= & \sum_{k=0}^{r}(-1)^{k}\left(\sum_{\left|\mu / \mu^{-}\right|=k} \operatorname{vs}_{\mu / \mu^{-}}(t) Q_{\mu^{-}}\right) \rightarrow\left(\sum_{\left|\lambda^{+} / \lambda\right|=r-k} \operatorname{sk}_{\lambda^{+} / \lambda}(t) P_{\lambda^{+}}\right) \\
= & \sum_{\lambda^{+}, \mu^{-}}(-1)^{\left|\mu / \mu^{-}\right|} \operatorname{vs}_{\mu / \mu^{-}}(t) \operatorname{sk}_{\lambda^{+} / \lambda}(t) P_{\lambda^{+} / \mu^{-}}
\end{aligned}
$$

For (23) and (24), the proof is the same as above. For (25), we used $e_{k}=P_{1^{k}}$, while for (26), we used (3) and (5). Equation (27) is obvious.

Proof of Theorem 4; Our first proof is along the lines of the preceding proofs of Theorems 2 and 3 .

$$
\begin{aligned}
P_{\lambda / \mu} \cdot q_{r} & =\left(Q_{\mu} \rightarrow P_{\lambda}\right) \cdot q_{r}=\sum_{\left(q_{r}\right)}\left(S\left(q_{r}{ }^{\prime \prime}\right) \rightarrow Q_{\mu}\right) \rightarrow\left(P_{\lambda} \cdot q_{r}{ }^{\prime}\right) \\
& =\sum_{k=0}^{r}\left(S\left(q_{k}\right) \rightarrow Q_{\mu}\right) \rightarrow\left(P_{\lambda} \cdot q_{r-k}\right) \\
& =\sum_{k=0}^{r}\left(\sum_{\tau \vdash k} c_{\tau}(t) P_{\tau} \rightarrow Q_{\mu}\right) \rightarrow\left(P_{\lambda} \cdot q_{r-k}\right) \\
& =\sum_{k=0}^{r}\left(\sum_{\tau \vdash k} c_{\tau}(t) Q_{\mu / \tau}\right) \rightarrow\left(P_{\lambda} \cdot q_{r-k}\right) \\
= & \sum_{k=0}^{r}\left(\sum_{\left|\mu / \mu^{-}\right|=k}\left(\sum_{\tau} c_{\tau}(t) f_{\mu^{-}, \tau}^{\mu}(t)\right) Q_{\mu^{-}}\right) \rightarrow\left(\sum_{\left|\lambda^{+} / \lambda\right|=r-k} \mathrm{hs}_{\lambda^{+} / \lambda}(t) P_{\lambda^{+}}\right) \\
= & \sum_{\lambda^{+}, \mu^{-}}(-1)^{\left|\mu / \mu^{-}\right|}(-t)^{\left|\tau / \mu^{-}\right|} \mathrm{vs}_{\mu / \tau}(t) \mathrm{sk}_{\tau / \mu^{-}} \mathrm{hs}_{\lambda^{+} / \lambda}(t) P_{\lambda^{+} / \mu^{-}} \cdot
\end{aligned}
$$

The only line that needs a comment is 33 . 
Substitute $y=-1 / t, \lambda=\mu, \mu=\mu^{-}$and $\nu=\tau$ into Theorem 7 . We get

$$
\sum_{\tau} \operatorname{sk}_{\tau / \mu^{-}}(t) \operatorname{vs}_{\mu / \tau}(t)(-1 / t)^{|\mu / \tau|}=\sum_{\sigma} t^{n(\sigma)-\left({ }^{\ell(\sigma)}\right)} f_{\tau, \mu^{-}}^{\mu}(t) \prod_{j=1}^{\ell(\sigma)}\left(-1 / t+t^{j-1}\right),
$$

which after a little arithmetic yields

$$
\sum_{\sigma} c_{\sigma} f_{\sigma, \mu^{-}}^{\mu}(t)=\sum_{\tau}(-1)^{|\mu / \tau|} t^{\left|\tau / \mu^{-}\right|} \mathrm{sk}_{\tau / \mu^{-}}(t) \mathrm{vs}_{\mu / \tau}(t)
$$

Our second proof uses Theorems 1,2 and 3 Recall from 9 that $q_{r}=\sum_{k=0}^{r}(-t)^{k} s_{r-k} e_{k}$. We have

$$
\begin{aligned}
P_{\lambda / \mu} \cdot q_{r}=P_{\lambda / \mu} \cdot\left(\sum_{k=0}^{r}(-t)^{k} s_{r-k} e_{k}\right)=\sum_{k=0}^{r}(-t)^{k}\left(P_{\lambda / \mu} s_{r-k}\right) e_{k} \\
=\sum_{k=0}^{r}(-t)^{k} \sum_{\sigma, \tau}(-1)^{|\mu / \tau|} \mathrm{vs}_{\mu / \tau}(t) \mathrm{sk}_{\sigma / \lambda}(t) P_{\sigma / \tau} e_{k} \\
=\sum_{\sigma, \tau, \mu^{-}, \lambda^{+}}(-t)^{\left|\tau / \mu^{-}\right|+\left|\lambda^{+} / \sigma\right|}(-1)^{|\mu / \tau|+\left|\tau / \mu^{-}\right|} \mathrm{vs}_{\mu / \tau}(t) \mathrm{sk}_{\sigma / \lambda}(t) \mathrm{sk}_{\tau / \mu^{-}}(t) \mathrm{vs}_{\lambda^{+} / \sigma}(t) P_{\lambda^{+} / \mu^{-}} \\
=\sum_{\tau, \mu^{-}, \lambda^{+}}(-1)^{\left|\mu / \mu^{-}\right|}(-t)^{\left|\tau / \mu^{-}\right|} \mathrm{vs}_{\mu / \tau}(t) \mathrm{sk}_{\tau / \mu^{-}}(t)\left(\sum_{\sigma}(-t)^{\left|\lambda^{+} / \sigma\right|} \mathrm{vs}_{\lambda^{+} / \sigma}(t) \mathrm{sk}_{\sigma / \lambda}(t)\right) P_{\lambda^{+} / \mu^{-}} \\
=\sum_{\tau, \mu^{-}, \lambda^{+}}(-1)^{\left|\mu / \mu^{-}\right|}(-t)^{\left|\tau / \mu^{-}\right|} \operatorname{vs}_{\mu / \tau}(t) \mathrm{sk}_{\tau / \mu^{-}}(t) \mathrm{hs}_{\lambda^{+} / \lambda}(t) P_{\lambda^{+} / \mu^{-}},
\end{aligned}
$$

where we used Lemma 5 in the final step.

Remark 12 It would be preferable to have a simpler expression for the polynomial

$$
d_{\lambda / \mu}(t)=\sum_{\nu}(-t)^{|\nu / \mu|} \operatorname{vs}_{\lambda / \nu}(t) \operatorname{sk}_{\nu / \mu}(t)
$$

i.e., one involving only the boxes of $\lambda / \mu$ in the spirit of $\mathrm{hs}_{\lambda / \mu}(t)$, so that we could write

$$
P_{\lambda / \mu} \cdot q_{r}=\sum_{\lambda^{+}, \mu^{-}}(-1)^{\left|\mu / \mu^{-}\right|} \mathrm{hs}_{\lambda^{+} / \lambda}(t) d_{\mu / \mu^{-}}(t) P_{\lambda^{+} / \mu^{-}}
$$

where the sum is over all $\lambda^{+} \supseteq \lambda, \mu^{-} \subseteq \mu$ such that $\left|\lambda^{+} / \lambda\right|+\left|\mu / \mu^{-}\right|=r$.

Toward this goal, we point out a hidden symmetry in the polynomials $d_{\lambda / \mu}(\mathrm{t})$. Writing $q_{r}$ as the sum $\sum_{k=0}^{r}(-t)^{k} e_{k} s_{r-k}$ before running through the second proof of Theorem 4 (i.e., applying Theorems 2 and 3 in the reverse order) reveals

$$
d_{\lambda / \mu}(t)=\sum_{\nu}(-t)^{|\lambda / \nu|} \operatorname{sk}_{\lambda / \nu}(t) \operatorname{vs}_{\nu / \mu}(t)
$$


Basic computations suggest some hint of a polynomial-product description for $d_{\lambda / \mu}(t)$,

$$
\begin{aligned}
& \square:-(t-1)^{2}(t+1)\left(t^{3}+t^{2}+t-1\right) \\
& :(t-1)^{2}(t+1)\left(t^{3}+t^{2}+t-1\right)^{2} \\
& : t(t-1)^{2}(t+1)\left(t^{2}+t-1\right)\left(t^{3}+t^{2}+t-1\right)^{2},
\end{aligned}
$$

but others suggest that such a description will not be tidy,

$$
\bigoplus:-t^{2}(t-1)^{2}(t+1)^{2}\left(t^{3}+t^{2}+t-1\right)\left(t^{7}+t^{6}+2 t^{5}-t^{3}-2 t^{2}-t+1\right)
$$

We leave a concise description of the $d_{\lambda / \mu}(t)$ as an open problem.

\section{References}

[AM11] Sami H. Assaf and Peter R. W. McNamara. A Pieri rule for skew shapes. J. Combin. Theory Ser. A, 118(1):277-290, 2011. With an appendix by Thomas Lam.

[Fu197] William Fulton. Young tableaux, volume 35 of London Mathematical Society Student Texts. Cambridge University Press, Cambridge, 1997.

[Kon] Matjaž Konvalinka. Skew quantum Murnaghan-Nakayama rule. To appear in J. Algebraic Combin., arXiv:1101.5250.

[LLS11] Thomas Lam, Aaron Lauve, and Frank Sottile. Skew Littlewood-Richardson rules from Hopf algebras. Int. Math. Res. Notices, 2011:1205-1219, 2011.

[LLT97] Alain Lascoux, Bernard Leclerc, and Jean-Yves Thibon. Ribbon tableaux, Hall-Littlewood functions, quantum affine algebras, and unipotent varieties. J. Math. Phys., 38(2):1041-1068, 1997.

[Mac95] I. G. Macdonald. Symmetric functions and Hall polynomials. Oxford Mathematical Monographs. The Clarendon Press Oxford University Press, New York, second edition, 1995.

[Mon93] Susan Montgomery. Hopf algebras and their actions on rings, volume 82 of CBMS Regional Conference Series in Mathematics. Published for the Conference Board of the Mathematical Sciences, Washington, DC, 1993.

[Sta99] Richard P. Stanley. Enumerative combinatorics. Vol. 2, volume 62 of Cambridge Studies in Advanced Mathematics. Cambridge University Press, Cambridge, 1999. 